ARTÍCULO ORIGINAL

\title{
ACTIVIDAD FOTOPROTECTORA DE UNA CREMA CON EXTRACTO ACUOSO LIOFILIZADO DE Lepidium meyenii (MACA) FRENTE A LA IRRADIACIÓN ULTRAVIOLETA EN PIEL DE RATONES
}

\author{
Malena Castañeda-Alarcón ${ }^{11, a}$, Carlos Bell-Cortez ${ }^{2, b}$, Julio Hidalgo-Ascensios ${ }^{(13, c}$, \\ Luis Moreno-Exebio (1D, 1,b \\ 1 Instituto Nacional de Salud, Lima, Perú. \\ 2 Universidad Nacional Mayor de San Marcos, Facultad de Farmacia y Bioquímica, Lima, Perú. \\ 3 Universidad Peruana Cayetano Heredia, LID-Facultad de Ciencias y Filosofía, Lima, Perú. \\ a Químico farmacéutico, magíster en Ciencias Farmacéuticas; ${ }^{\mathrm{b}}$ químico farmacéutico, doctor en Farmacia y Bioquímica; ${ }^{\mathrm{c} b i o ́ l o g o .}$ \\ El presente estudio forma parte de la tesis: Castañeda-Alarcón M. Evaluación de la actividad fotoprotectora in vitro y efecto \\ fotoprotector in vivo de una formulación a base de extracto acuoso liofilizado de Lepidium meyenii (MACA) [tesis de maestría]. \\ Lima: Facultad de Farmacia y Bioquímica, Universidad Nacional Mayor de San Marcos; 2019.
}

\section{RESUMEN}

Objetivos: Evaluar la actividad fotoprotectora de una crema con extracto acuoso liofilizado de maca (ELM) frente a la irradiación ultravioleta (UV) en la piel de ratones. Materiales y métodos: Se realizó un estudio experimental en 35 ratones $\mathrm{BALB} / \mathrm{c}$. Los tratamientos fueron aplicados por vía tópica en el dorso de los animales y posteriormente irradiados con rayos ultravioleta $B$, para luego medir el grosor en micras $(\mu \mathrm{m})$ de muestras histológicas de la piel de los ratones. Se asignaron siete grupos divididos en no irradiado: blanco (G1) e irradiados con luz UV: sin tratamiento (G2); con protector solar comercial con factor de protección solar (FPS) 30 (G3); crema (placebo) (G4); ELM al 15\% en agua (G5); ELM al 5\% en crema (G6); y ELM al 15\% en crema (G7). Se determinó el FPS in vitro, mediante el método de Mansur. Se realizaron las lecturas de las absorbancias en un espectrofotómetro ultravioleta-visible (UV-VIS) y se determinaron los FPS para las siguientes formulaciones: ELM al 5\% en crema, benzofenona-4 (BZF-4) y bloqueador solar comercial FPS 30. Resultados: El grosor de piel de ratón en micras $(\mu \mathrm{m})$ fue de 27,28 en G2; 18,31 en G3; 27,33 en G4; 19,51 en G5 y 18,04 en G6; no hubo diferencia significativa entre el grupo no expuesto a la radiación (G1) y el grupo ELM al 15\% en crema (G7), ambos presentaron los menores grosores (12,76 y $14,20 \mu \mathrm{m}$, respectivamente). El FPS de ELM al $15 \%$ en crema fue 5,480 \pm 0,020. Conclusiones: La formulación con ELM en crema presentó actividad fotoprotectora frente a la irradiación UV, los alcaloides fueron los componentes fitoquímicos mayormente encontrados y la formulación fue compatible con el activo (ELM).

Palabras clave: Efectos de la Radiación; Lepidium; Factor de Protección Solar; Protectores Solares; Fitoterapia (fuente: DeCS BIREME).

\section{PHOTOPROTECTIVE ACTIVITY OF A CREAM CONTAINING LYOPHILIZED AQUEOUS EXTRACT OF Lepidium meyenii (MACA) AGAINST ULTRAVIOLET IRRADIATION ON MOUSE SKIN}

Citar como: Castañeda-Alarcón M Bell-Cortez C, Hidalgo-Ascensios J, Moreno-Exebio L. Actividad fotoprotectora de una crema con extracto acuoso liofilizado de Lepidium meyenii (MACA) frente a la irradiación ultravioleta en piel de ratones. Rev Peru Med Exp Salud Publica. 2021;38(3):43441. doi: https://doi.org/10.17843/ rpmesp.2021.383.7420.

Correspondencia: Malena Liuva Castañeda Alarcón; Av. Defensores del Morro 2268, Centro Nacional de Control de Calidad del Instituto Nacional de Salud, Chorrillos; mcastaneda@ins.gob.pe

Recibido: $24 / 02 / 2021$ Aprobado: 08/09/2021 En línea: 31/09/2021

\section{ABSTRACT}

Objectives: To evaluate the photoprotective activity of a cream with lyophilized aqueous extract of maca (LEM) against ultraviolet (UV) irradiation in the skin of mice. Materials and methods: An experimental study was carried out on $35 \mathrm{BALB} / \mathrm{c}$ mice. Treatment was applied topically on the dorsum of the animals, which were subsequently irradiated with ultraviolet B rays, and then we measured the thickness in microns ( $\mu \mathrm{m})$ of histological samples of the skin of the mice. Seven groups were assigned, divided into non-irradiated: white (G1) and irradiated with UV light: no treatment (G2); with commercial sunscreen with sun protection factor (SPF) 30 (G3); cream (placebo) (G4); LEM at 15\% in water (G5); LEM cream at 5\% (G6); and LEM cream at $15 \%$ (G7). In vitro SPF was determined using the Mansur method. Absorbance readings were taken in an ultravioletvisible spectrophotometer (UV-VIS) and SPFs were determined for the following formulations: LEM cream at $5 \%$, benzophenone-4 (BZF-4) and commercial sunscreen SPF 30. Results: Mouse skin thickness in microns ( $\mu \mathrm{m})$ was 27.28 in G2; 18.31 in G3; 27.33 in G4; 19.51 in G5 and 18.04 in G6. There was no significant difference between the group not exposed to radiation (G1) and the 15\% LEM cream group (G7), both had the lowest thicknesses (12.76 and $14.20 \mu \mathrm{m}$, respectively). The SPF of LEM cream at $15 \%$ was $5.480 \pm 0.020$. Conclusions: The formulation with LEM cream showed photoprotective activity against UV irradiation, alkaloids were the phytochemical components mostly found and the formulation was compatible with the active principle (LEM).

Keywords: Radiation Effects; Lepidium; Sun Protection Factor; Sunscreening Agents; Phytotherapy (source: MeSH NLM). 


\section{INTRODUCCIÓN}

En el mundo se ha evidenciado el incremento de la radiación ultravioleta (UV) como consecuencia inmediata del adelgazamiento de la capa de ozono ${ }^{(1,2)}$, lo que constituye una amenaza para el ser humano por el alto potencial cancerígeno producto de su exposición ${ }^{(3)}$. La radiación UV acumulativa incrementada y el grado de sensibilidad según el tipo de piel están asociados al cáncer no melanómico y al melanoma cutáneo, respectivamente ${ }^{(4,5)}$.

Los cambios climáticos y medioambientales han ocasionado una disminución de la capa de ozono, favoreciendo una mayor exposición a la radiación solar ${ }^{(6)}$. El Centro Nacional de Epidemiología, Prevención y Control de Enfermedades indicó a través de reportes hospitalarios que el cáncer de piel $(10,8 \%)$ ocupa el tercer lugar en frecuencia después del cáncer de cérvix $(18,6 \%)$ y de estómago $(11,1 \%)$, con relación al total de cánceres registrados ${ }^{(7)}$.

Una alternativa de protección es el uso de fórmulas fotoprotectoras con filtros UV, que interfieren directamente con la radiación solar a través de la absorción, reflexión o dispersión de energía ${ }^{(2)}$. Estos filtros se clasifican como químicos (orgánicos) caracterizados por absorber los rayos UV y filtros físicos (inorgánicos) cuyo mecanismo de acción se basa en la reflexión y dispersión de la luz $\mathrm{UV}^{\left({ }^{(8)}\right.}$. Se sabe que algunos filtros químicos se absorben sistémicamente, otros dañan a los corales de los arrecifes, como la oxibenzona ${ }^{(9)}$, o pueden resultar fotoinestables como la avobenzona ${ }^{(10)}$.

Ciertos extractos vegetales elaborados a partir de metabolitos de plantas pueden utilizarse como filtros solares, gracias a su capacidad de absorber diferentes radiaciones ${ }^{(11)}$, combinándose con otros componentes de síntesis o pigmentos minerales para optimizar la eficacia fotofiltrante ${ }^{(10,12)}$.

Los extractos vegetales usados para filtración solar deben cumplir con características específicas: presentar una absorción de la radiación que oscile entre $290-400 \mathrm{~nm}^{(13)}$, ser probadas en condiciones normales de uso y fabricación, ser compatibles con los excipientes y con el material de acondicionamiento de los productos utilizados en las líneas solares $\mathrm{y}$, finalmente, no ser tóxicos ${ }^{(2)}$.

La maca (Lepidium meyenii o Lepidium peruvianum Chacon) es una planta originaria de los Andes centrales del Perú, resistente a granizadas, heladas y sequías prolongadas ${ }^{(14)}$; se cultiva desde la época incaica en altitudes comprendidas entre los 3800 y los $4500 \mathrm{~m} \mathrm{s.} \mathrm{n.} \mathrm{m.}{ }^{(15)}$. La evidencia indica que el aumento en la producción de alcaloides está relacionado con la altitud y con la exposición a la radiación solar, tal como se descrito en Meconopsis quintuplinervia, planta originaria de China ${ }^{(16)}$, y en Árnica montana que, con la altitud incrementó sus derivados de ácido cafeido ${ }^{(17)}$. Es probable que los organismos que viven en estas altitudes desarrollen mecanismos de protección contra la sobreexposición a la radiación UV. Exis-

\section{MENSAJES CLAVE}

Motivación para realizar el estudio: El efecto de la radiación ultravioleta (UV) produce patologías en la piel que pueden ocasionar cáncer. Es necesario buscar alternativas en la fitoterapia que coadyuven a la fotoprotección. La maca es una planta que crece en las alturas a elevada radiación, por ello podría tener metabolitos en su composición que le brinde protección contra la radiación UV.

Principales hallazgos: El empleo tópico de una crema formulada con Lepidium meyenni (maca) presentó actividad fotoprotectora y compatibilidad en la formulación.

Implicancias: Promover el uso de la maca como alternativa coadyuvante para la fotoprotección, probablemente debido a la presencia de alcaloides y continuar buscando alternativas de filtros naturales y estables.

ten estudios experimentales que reportan alguna protección frente a la irradiación UV a partir de la aplicación tópica de extracto de maca $^{(12,18)}$.

Se requieren formulaciones fotoprotectoras para prevenir los efectos dañinos de la radiación UV sobre la piel con activos estables e inocuos o que sirvan de coadyuvantes para optimizar la acción de los filtros químicos. Existe evidencia previa del uso de extractos de plantas fotoprotectoras, por ello es posible plantear el uso del extracto de maca con fines fotoprotectores de forma que pueda utilizarse en formulaciones fitoterapéuticas. Por tal motivo éste estudio tiene por objetivo evaluar la actividad fotoprotectora de una formulación tópica de maca mediante irradiación UV en la piel de ratones, así como la determinación del factor de protección solar (FPS) in vitro.

\section{MATERIALES Y MÉTODOS}

\section{Diseño}

Se realizó un estudio experimental utilizando extracto acuoso liofilizado de Lepidium meyenii «maca» (ELM) aplicado tópicamente sobre la piel de ratones. La preparación del extracto acuoso y la marcha fitoquímica se realizó en el Laboratorio de Química Analítica y Farmacognosia de la Facultad de Farmacia y Bioquímica de la Universidad Nacional Mayor de San Marcos (UNMSM). La liofilización y el bioensayo se realizó en el Laboratorio de Microbiología y Virología, de la Universidad Peruana Cayetano Heredia (UPCH). La formulación, evaluación del FPS y evaluación fisicoquímica se realizó en el Laboratorio de Fisicoquímica, del Centro Nacional de Control de Calidad (CNCC) del Instituto Nacional de Salud (INS). La evaluación histológica se 
realizó en el Instituto de Patología, de la Facultad de Medicina de la UNMSM.

\section{Animales \\ Se usaron 35 ratones machos de cepa BALB/c de $26 \pm 2 \mathrm{~g}$ de peso promedio, de aproximadamente 10 semanas de edad que fueron adquiridos del bioterio de la UPCH. Los rato- nes se colocaron en siete jaulas, a razón de cinco por jaula. Fueron puestos en un periodo de cuarentena de cinco días, antes de iniciar el ensayo, con fines de adaptación fisiológi- ca y conductual. Las condiciones del ensayo fueron las si- guientes: temperatura de $20-25^{\circ} \mathrm{C}$, humedad de $50-70 \%$, y fotoperiodo de 12 horas de luz y 12 horas de oscuridad. Los animales consumieron alimentos balanceados, provenientes de la Universidad Nacional Agraria La Molina, y agua ad $l i-$ bitum. Se cumplieron con todas las directrices descritas en la Guide for the care and use of Laboratory animals ${ }^{(19)}$ y la «Guía de manejo y cuidado de animales de laboratorio: ratón» ${ }^{(20)}$. \\ Preparación del extracto acuoso liofilizado de Lepidium meyenii}

Las raíces de Lepidium meyenii variedad amarilla se recolectaron de la zona andina de Pachacayo (3600 m s. n. m.) distrito de Canchaylla, provincia de Jauja, en la región Junín, en mayo de 2014. La identificación taxonómica se realizó en el Museo de Historia Natural, de la UNMSM (Constancia N. ${ }^{\circ}$ 57-USM-2014). Para preparar el ELM se pesaron $500 \mathrm{~g}$ de raíces de maca (previamente secadas y pulverizadas) y se cocieron con siete litros de agua desionizada por 60 minutos. El filtrado se guardó a $-20^{\circ} \mathrm{C}$ y, posteriormente, se liofilizó.

\section{Marcha fitoquímica}

Se realizó la marcha fitoquímica para determinar los compuestos fitoquímicos mediante reacciones de coloración o precipitación ${ }^{(12)}$.

\section{Formulación}

La solubilidad se determinó usando los siguientes solventes: metanol, alcohol etílico y agua, ordenados de menor a mayor polaridad. Se formularon emulsiones aceite/agua $(\mathrm{O} / \mathrm{W})$ a las que se incorporó el ELM en dos concentraciones de 5\% y $15 \%{ }^{(21)}$.

\section{Actividad fotoprotectora - bioensayo}

Para determinar la actividad fotoprotectora de la formulación con ELM se empleó el modelo biológico ${ }^{(22)} \mathrm{de}$ la incidencia de irradiación UV en la piel de ratón ${ }^{(18,23)}$. Se trabajó con siete grupos de ratones, cinco por grupo, que fueron tratados por vía tópica. El primer grupo (G1) fue el único que no estuvo expuesto a la radiación UV. Los demás grupos estuvieron expuestos y se clasificaron de siguiente forma: sin tratamiento (G2); protección solar comercial con
FPS 30 (G3); placebo (formulación sin ELM) (G4); ELM al $15 \%$ en agua (G5); ELM al 5\% en crema (G6), y ELM al 15\% en crema (G7). Se trazó un círculo de aproximadamente de $2 \mathrm{~cm}$ de diámetro en la superficie dorsal de cada animal para posteriormente depilarlos 24 horas antes del inicio de la irradiación UV. Se dejó reposar a los ratones para observar alguna alteración a nivel dermal (irritación, eritema o edema). El volumen de aplicación por cada tratamiento fue de $100 \mathrm{uL}$ por área depilada expuesta.

Los ratones se anestesiaron con ketamina (40 mg/kg) y xilacina $(15 \mathrm{mg} / \mathrm{kg})$ minutos antes de la irradiación en la cámara de exposición. Se colocó cada ratón a $15 \mathrm{~cm}$ de la fuente de irradiación UV durante 30 minutos. La irradiación se realizó una vez al día durante tres días consecutivos (equipo: Spectroline, LongLife filtro n. $^{\circ}$ 1575586, NY, EE. UU.), siendo $3,3 \mathrm{~mW} / \mathrm{cm}^{2}$ la cantidad de energía de UV expuesta sobre la piel y medida por medio de un radiómetro (UDT 371 instrumentos ópticos medidor de potencia, modelo \# 268 UVC; Thorlabs, Newton, Nueva Jersey, EE. UU.).

Para la evaluación histológica se sacrificaron los animales por sobredosificación de anestésico, dos horas después de la última exposición. Por cada animal se seccionó una muestra de piel de $2 \mathrm{~cm}$ de diámetro, el cual se colocó en formol al $4 \%$ para realizar una lectura histológica. Los fragmentos de piel se procesaron por la técnica de inclusión en parafina y coloración con hematoxilina y eosina para su estudio histopatológico. Las observaciones se llevaron a cabo utilizando un microscopio óptico Nikon eclipse Ci (Japón) realizando mediciones por secciones entre el estrato granulosum y la unión dermoepidérmica ${ }^{(18)}$.

\section{Determinación del factor de protección solar}

Se pesó $1 \mathrm{~g}$ de la formulación con ELM al 15\% y se realizaron diluciones hasta obtener una concentración final de $0,2 \mathrm{mg} / \mathrm{mL}$. Se procedió igual para la formulación con benzofenanona-4 (BZF-4) al 1,5\% y para el bloqueador solar del mercado. Las absorbancias de las soluciones fueron determinadas mediante un espectrofotómetro UV Jasco V-650 Spectrophotometer (Japón), en el rango de 290 a $320 \mathrm{~nm}$ (Tabla 1), con intervalos de $5 \mathrm{~nm}$ utilizando una cubeta de cuarzo de $1 \mathrm{~cm}$. Los análisis fueron

FPS espectrofotométrico $=$ FC. ${ }^{320} \sum_{290} \times \operatorname{EE}(\lambda) \times \mathrm{I}(\lambda) \times$ ABS $(\lambda)$

Dónde FPS: factor de protección solar; FC: 10 (factor de corrección); $\mathrm{EE}(\lambda)$ : efecto $\mathrm{ABS}$ de la radiación de longitud de onda $\lambda$; I $(\lambda)$ : intensidad del sol en la longitud de onda $\lambda$; $\operatorname{ABS}(\lambda)$ : absorbancia de la solución en la longitud de onda $\lambda$. La relación entre el efecto eritemogénico y la intensidad de la radiación de cada longitud de onda $(\operatorname{EE}(\lambda) \times \mathrm{I}(\lambda))$ es una constante determinada por Sayre et al. (30) (Tabla 1). 
Tabla 1. Relación entre el efecto eritemogénico (EE) versus la intensidad de radiación (I) conforme la longitud de onda $(\lambda)$ constante determinado por Sayre et al.

\begin{tabular}{lc}
\hline Longitud de onda $\boldsymbol{\lambda}(\mathbf{n m})$ & EE x I (normalizado) \\
\hline 290 & 0,0150 \\
295 & 0,0817 \\
300 & 0,2874 \\
305 & 0,3278 \\
310 & 0,1864 \\
315 & 0,0839 \\
320 & 0,0180 \\
Total & 1 \\
\hline
\end{tabular}

EE: efecto eritemogénico de la radiación de longitud de onda; I: intensidad del sol en la longitud de onda. Fuente: Sayre et al ${ }^{(30)}$.

realizados por triplicado y el FPS fue calculado de acuerdo con la ecuación matemática desarrollada por Mansur et al. ${ }^{(24)}$

\section{Evaluación de las características fisicoquímicas}

Se evaluó el aspecto, color y olor de la formulación con ELM al 15\% y al 5\% mediante inspección sensorial, visual y olfativa en muestras almacenadas a temperatura ambiente, evitando el contacto con la luz solar. Para la determinación del pH, se utilizó potenciómetro $691 \mathrm{pH}$ Meter Metrohm (Suiza), electrodo de vidrio combinado Metrohm 6.0228.00 (Suiza), se usó el capítulo $<791>\mathrm{pH}$, USP 38 y soluciones buffers ( $\mathrm{pH}$ 4,00 y pH 7,00). Para la determinación de separación de fases, se centrifugaron las muestras en el equipo EVA21 Hettich Zentrifugen (Alemania) a $3000 \mathrm{rpm}$ por 30 minutos ${ }^{(25)}$. Para el ensayo de viscosidad se usó Brokfiels DV-II+Viscosimeter (MA 02346 Estados Unidos) se midió a $25^{\circ} \mathrm{C}$ de temperatura. Se evaluó a las 24 horas, 90, 180 y 240 días.

\section{Análisis estadístico}

Para la evaluación del grosor de la piel de ratón en micras, se realizó la prueba de normalidad de Shapiro Wilk para cada grupo de tratamiento incluyendo los controles positivos y negativos, en el análisis intergrupos se utilizó la prueba no paramétrica de Kruskal Wallis debido a que las varianzas intergrupos eran desiguales (prueba de Bartlett p=0,010), se utilizó la prueba de Dunn para realizar inferencias simultáneas en parejas. Asimismo, los resultados del factor de protección solar fueron expresados con la media y desviación estándar. Se asumió un nivel de significancia de 0,05 para todas las pruebas estadísticas. Todos los análisis se realizaron con el programa estadístico Stata 15.

\section{Aspectos éticos}

Este estudio fue aprobado por el Comité Institucional de Ética en Investigación del Instituto Nacional de Salud (CIEIINS) (Código N. ${ }^{\circ}$ OT-047-14) y por el Comité de Ética para el uso de Animales en Investigación del Instituto Nacional de Salud (CIEA-INS). El método de eutanasia empleado fue sobredosificación de anestésico, un método aceptable para roedores descrito en Guide for the Care and Use of Laboratory Animals ${ }^{(19)}$.

\section{RESULTADOS}

En el estudio fitoquímico de ELM, se encontraron, principalmente, alcaloides o compuestos nitrogenados, y en menor proporción azúcares y compuestos fenólicos, no se encontró presencia de taninos, quinonas, esteroles y flavonoides (Tabla 2). Respecto a la solubilidad, el ELM fue totalmente soluble en agua, parcialmente en alcohol etílico e insoluble en metanol.

En la evaluación de la actividad fotoprotectora de la maca en ratones, los grupos en los que no se usó tratamiento (G2) o en los que se usó crema sin ELM (G4) la mediana del grosor de la piel fue mayor $(27,28 \mu \mathrm{m}$ y $27,33 \mu \mathrm{m}$, respectivamente) en comparación al grupo que no estuvo expuesto a la radiación (G1) o al grupo que utilizó algún protector solar comercial (G3) y a los que usaron el ELM en las formulaciones. Entre los grupos de intervención, la mediana de menor grosor se encontró en el que utilizó crema con ELM al 15\% (G7) (14,2 RI: 10,42-19,27) (Tabla 3, Figura 1).

Para las inferencias por pares, en el grupo no expuesto a la radiación (G1) se encontraron diferencias estadísticamente significativas en comparación con los grupos expuestos a la radiación sin tratamiento $(\mathrm{G} 2)(\mathrm{p}<0,001)$, con protector solar con FPS $30(p=0,041)$, crema con ELM $(p<0,001)$, agua con ELM 15\% ( $\mathrm{p}=0,005)$ y crema con ELM 5\% $(\mathrm{p}=0,044)$ (Tabla 4).

Para el G3 solo se encontró diferencia significativa con $\mathrm{G} 4$ ( $\mathrm{p}=0,050)$. En los demás casos, todas las fórmulas ofrecen fotoprotección con variaciones no detectables significativamente en el grosor de la piel. En el caso de G4 se encontró

Tabla 2. Compuestos presentes en extracto acuoso liofilizado de Lepidium meyenni (ELM)

\begin{tabular}{lcc}
\hline Grupo de metabolito & Reactivo utilizado & ELM \\
\hline Taninos & Gelatina & - \\
Proteínas (aminoácidos) & Ninhidrina & - \\
Compuestos fenólicos & Tricloruro de hierro & + \\
Alcaloides o compuestos & Dragendorff & ++ \\
nitrogenados & Mayer & ++ \\
Alcaloides & Borntrager & - \\
Quinonas & Lieberman & - \\
Esteroles & Molish & + \\
Azúcares & Shinoda & - \\
Flavonoides & & \\
\hline
\end{tabular}

$(-)$ ausencia; $(+)$ poca cantidad; $(++)$ regular cantidad

Se observa la evaluación cualitativa, según la reacción de color o precipitado. 
Tabla 3. Distribución del grosor de la piel de los ratones según grupo

\begin{tabular}{llcc}
\hline & Grupo & \multicolumn{2}{c}{ Grosor de la piel $(\boldsymbol{\mu m})$} \\
\cline { 3 - 4 } & Mediana & $\begin{array}{c}\text { Rango } \\
\text { intercuartílico }\end{array}$ \\
\hline G1 & No expuesto a radiación & 12,76 & $9,9-15,47$ \\
G2 & Sin tratamiento & 27,28 & $22,84-33,04$ \\
G3 & Protector solar comercial SFP 30 & 18,31 & $18,26-18,42$ \\
G4 & Crema sin ELM & 27,33 & $22,47-29,69$ \\
G5 & Agua + ELM 15\% & 19,51 & $14,59-26,61$ \\
G6 & Crema + ELM 5\% & 18,04 & $15,32-22,31$ \\
G7 & Crema + ELM 15\% & 14,2 & $10,42-19,27$ \\
\hline
\end{tabular}

ELM: extracto acuoso liofilizado de maca

diferencia significativa con G6 ( $\mathrm{p}=0,047)$ y G7 $(\mathrm{p}<0,001)$, y no se encontró diferencia significativa con $\mathrm{G} 5(\mathrm{p}=0,088)$. G5 presenta diferencia significativa con $(\mathrm{G} 7)(\mathrm{p}=0,011)$. Finalmente, en G6 no se encontró diferencia significativa con G7, lo cual indica que con relación al grosor de la piel la fotoprotección de la fórmula y la concentración al 5\% y al $15 \%$ no tendría diferencia significativa (Tabla 4 ).

En la determinación del FPS in vitro calculado en nanómetros $(\mathrm{nm})$ para la formulación de crema con ELM al 15\% fue de 5,480 $\pm 0,020$, para BZF- 4 al 1,5\% fue de 6,854 $\pm 0,001$ y para el bloqueador solar del mercado fue de 11,504 $\pm 0,027$.

En la evaluación fisicoquímica de la formulación se observó compatibilidad entre el ELM y la crema (base), lo que dio como resultado un preparado de aspecto uniforme, sin cambios de textura, color y olor, y no se evidenció floculación, coagulación, precipitación, coalescencia ni granulado. Las cremas con ELM presentaron un olor suigéneris propio de la especie. Al ser centrifugado no se observó separación de fases o cambios de textura del producto. El pH se encontró dentro del rango aceptable para uso tópico (Tabla 5).

\section{DISCUSIÓN}

En el presente estudio se observa que la crema con ELM al $15 \%$ presentó actividad fotoprotectora en la piel de ratones irradiados con rayos UV, la formulación también presentó FPS in vitro y se determinó que los principales componentes del ELM eran los alcaloides.

El modelo biológico proporciona información sobre el daño que causa la radiación UV sobre la piel de ratón irradia$\mathrm{do}^{(22)}$. La exposición a la radiación UV sin protección produce un aumento del grosor epidérmico (hiperplasia) y un aumento del número de células de quemadura solar ${ }^{(2)}$. Gonzales-Castañeda ${ }^{(18)}$ encontró que los extractos de hojas de maca amarilla, negra y roja disminuyen el grosor epidérmico, evita la infiltración leucocitaria y la generación de queratinocitos atípicos muy similar al grupo control. En el presente estudio se observaron resultados similares, ya que no se encontró diferencia significativa entre el grupo control (G1) no expuesto a la radiación y el grupo (G7) crema con ELM al 15\%, esto podría explicarse porque la protección otorgada por el grupo de mayor concentración del principio activo es bastante similar a la no exposición y, por lo tanto, no habría cambios significativos de la unión dermoepidérmica.

Para el grupo sin tratamiento con irradiación (G2) se encontraron diferencias significativas con el grupo con protector solar comercial (G3), crema con ELM al 5\% (G6) y crema con ELM al 15\% (G7), esto puede explicarse porque las fórmulas contienen el principio activo de ELM o el filtro solar de la formulación comercial; sin embargo, en los grupos de crema sin ELM (G4) y agua con ELM al 15\% (G5) no se reporta diferencia significativa, podría ser porque en G4 tampoco hay un principio activo que brinde fotoprotección y en el G5, a pesar de haber el ELM al 15\%, el vehículo es agua, el cual no sería el más adecuado para su aplicación tópica por su menor tiempo de fijación en la piel ${ }^{(26)}$, que el vehículo crema. En el grupo crema sin ELM (G4) se encontró diferencia significativa con las formulaciones de crema con ELM al 5\% y crema con ELM al $15 \%$, esto se explicaría por la existencia de efecto fotoprotector en las dos concentraciones.

Tabla 4. Valores de p obtenidos con la prueba de Dunn para comparar el grosor de piel según grupos.

\begin{tabular}{lcccccc}
\hline Grupos & G1 & G2 & G3 & G4 & G5 & G6 \\
\hline G2 & $<0,001$ & & & & & \\
G3 & 0,041 & 0,034 & & & & \\
G4 & $<0,001$ & 0,423 & 0,050 & & & \\
G5 & 0,005 & 0,059 & 0,309 & 0,088 & & \\
G6 & 0,044 & 0,031 & 0,488 & 0,047 & 0,296 & \\
G7 & 0,323 & $<0,001$ & 0,076 & $<0,001$ & 0,011 & 0,081 \\
\hline
\end{tabular}

ELM: extracto acuoso liofilizado de maca; G1: blanco (no expuesto a radiación); G2: sin tratamiento; G3: con protector solar comercial con factor de protección solar 30; G4: crema sin ELM (placebo); G5: ELM al 15\% en agua; G6: ELM al 5\% en crema; G7: ELM al 15\% en crema 
Tabla 5. Análisis fisicoquímico del extracto acuoso liofilizado de Lepidium meyenii al 15\% en crema

\begin{tabular}{|c|c|c|c|c|c|c|}
\hline \multirow{2}{*}{ Características } & \multirow{2}{*}{ Parámetro } & \multirow{2}{*}{ Especificaciones } & \multicolumn{4}{|c|}{ Resultados } \\
\hline & & & 24 horas & 90 días & 180 días & 240 días \\
\hline \multirow{4}{*}{ Organolépticas } & Homogeneidad & Crema homogénea & $\begin{array}{l}\text { Homogéneo } \\
\text { (ausencia de } \\
\text { grumos) }\end{array}$ & $\begin{array}{l}\text { Homogéneo } \\
\text { (ausencia de } \\
\text { grumos) }\end{array}$ & $\begin{array}{l}\text { Homogéneo } \\
\text { (ausencia de } \\
\text { grumos) }\end{array}$ & $\begin{array}{l}\text { Homogéneo } \\
\text { (ausencia de } \\
\text { grumos) }\end{array}$ \\
\hline & Sensación al tacto & Suave al tacto & Suave al tacto & Suave al tacto & Suave al tacto & Suave al tacto \\
\hline & Color & Amarillo pardo & Amarillo pardo & Amarillo pardo & Amarillo pardo & Amarillo pardo \\
\hline & Olor & Característico & Suigéneris & Suigéneris & Suigéneris & Suigéneris \\
\hline \multirow[b]{2}{*}{ Fisicoquímicas } & $\mathrm{pH}\left(25^{\circ} \mathrm{C}\right)$ & $4,5-7,00$ & 4,73 & 4,79 & 4,85 & 4,96 \\
\hline & $\begin{array}{l}\text { Viscosidad (cps) } \\
\left(25^{\circ} \mathrm{C}\right)\end{array}$ & $8001-12000$ & 10590 & 10562 & 10534 & 10508 \\
\hline
\end{tabular}

cps: centipoise equivalente a un milipascal segundo.

Respecto al FPS usando el método de Mansur para la determinación del FPS in vitro, diversos estudios encontraron un FPS por debajo de 3 como lo reporta Costa et al. ${ }^{(13)}$ con un FPS de 2,23 en una formulación con extracto de Marcetia taxifolia al 20\%. Alayo et al. ${ }^{(21)}$ obtuvieron un FPS de 2,29 en una crema fotoprotectora de tipo $\mathrm{O} / \mathrm{W}$ con extracto hidroalcohólico de las hojas de Piper aduncum. Inocente et al. ${ }^{(27)}$ re- portaron en una formulación con extracto de camu camu al $15 \%$ un FPS de 2,667 $\pm 0,044$ y para la loción placebo un FPS de $0,589 \pm 0,057$, respectivamente. Estos resultados podrían explicarse porque los valores bajos de FPS son consecuencia de la baja concentración de las moléculas con capacidad para absorber la radiación UV (cromóforos) ${ }^{(27)}$. Asimismo, podrían considerarse en la categoría de FPS bajo, como lo

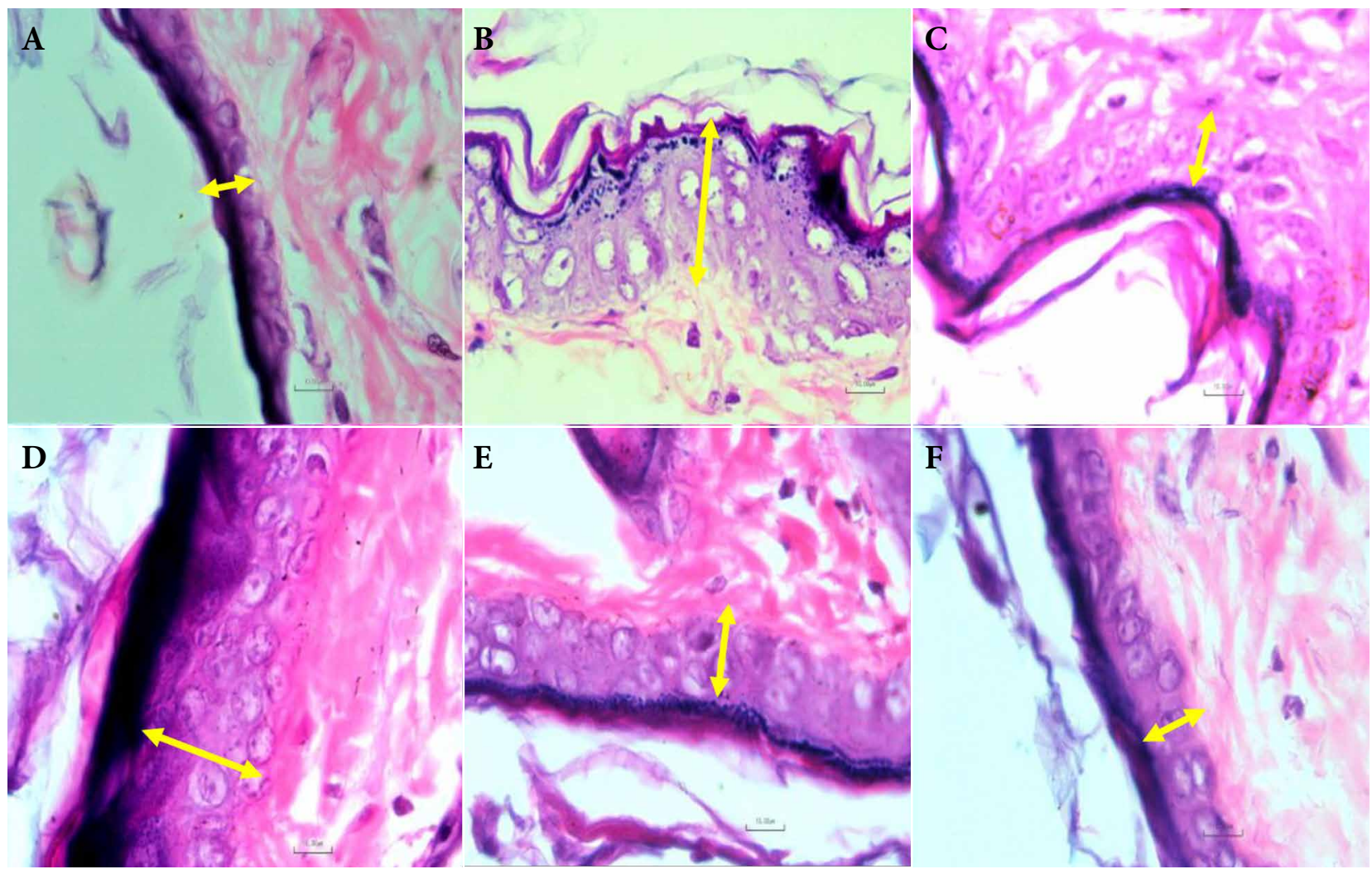

Figura 1. Microfotografía de la piel de ratones expuestos a radiación UV. Las flechas amarillas indican el grosor epidérmico evaluado (magnitud 400×). Se midió desde la unión dermoepidermica hasta el estrato granuloso, sin incluir el estrato corneo. Técnica de coloración: eosina-hematoxilina.

A) Blanco; sin irradiación ultravioleta (UV) y sin tratamiento. El grosor de la unión dermoepidérmica es menor en relación al control. B) Control; con irradiación UV y sin tratamiento. Se observa hiperplasia epidérmica, visto como un aumento del grosor dermoepidérmico, siendo este mayor que el blanco. C) Protector solar comercial, con irradiación UV. Se observa ligero incremento del grosor dermoepidérmico en relación al blanco. D) Crema sin extracto acuoso liofilizado de maca (ELM), con irradiación UV. El grosor dermoepidérmico es similar al control. E) Crema con ELM al 5 \%, con irradiación UV Se observa que el grosor de la unión dermoepidérmica es menor que crema + ELM al 15\%. F) Crema con ELM al 15\%, con irradiación UV. Se observa que el efecto de hiperplasia epidérmica disminuye, el grosor dermoepidérmico es similar al blanco. 
indica la Australian Regulatory Guidelines for Sunscreens del $2019^{(28)}$.

Este estudio encontró en la formulación (crema con ELM al $15 \%)$ un FPS de 5,480 $\pm 0,020$. Estos resultados son diferentes a lo reportado por Prudencio Quiroz y Bustamante Arroyo ${ }^{(12)} \mathrm{en}$ su formulación con extracto hidroglicólico de Lepidium meyenii (base + filtro solar 5\% + maca 10\%) con un FPS de 8,354 $\pm 0,003$, cabe mencionar que su formulación utilizó un filtro solar (benzofenona-3) al 5\% con un FPS de 4,960 $\pm 0,001$. Los resultados entre ambos estudios difieren debido a los diferentes tipos de extractos de maca utilizados.

Respecto a las concentraciones de extracto en las formulaciones, Soares et al. ${ }^{(11)}$ reportaron un FPS de 5,05 en una formulación con extracto de propóleo al $20 \%$ y $40 \%$, en esta última encontró valores de FPS sobre 10. Se puede inferir que, para llegar a FPS superiores a 10, se deben usar concentraciones superiores al $20 \%$ de los extractos de plantas que actúan como filtros solares.

Zhou et al. ${ }^{(29)}$ encontraron principalmente alcaloides, glucosinolatos y macaenos en los extractos de Lepidium meyenii, de manera similar al presente estudio, cuyos componentes mayoritarios fueron también los alcaloides. Asimismo, estudios realizados anteriormente han aislado tres tipos de alcaloides de las raíces de maca: alcaloides imidazólicos de Lepidiline A y B y la Macaridina (derivado bencilado1,2-dihidro-N-hidroxipiridina) ${ }^{(15)}$, Yang et al. ${ }^{(16)}$ reportaron que el contenido total de alcaloides de Meconopis uintuplinervia aumenta con la altitud. Spitaler et al. ${ }^{(17)}$ indicaron que los metabolitos secundarios de Árnica Montana estaban relacionados con la altitud, resultados que se discuten por la captación de radicales de compuestos fenólicos y su importancia para la vida vegetal en ambientes con radiación UV elevada, esto podría indicar que los alcaloides son los responsables de la actividad fotoprotectora.

Sobre el control de la calidad de las formulaciones con ELM al 15\% se observó que las propiedades macroscópicas reflejaron una correcta interacción entre los componentes, una elevada compatibilidad entre el ELM y todos los excipientes de la formulación, la base de nuestra formulación fue una emulsión $\mathrm{O} / \mathrm{W}$, base que tiene mayor cantidad de medio acuoso. Existe referencia que los alcaloides de la maca se combinan con los ácidos dando lugar a la formación de sales de alcaloides, estos son cristalizables y solubles en agua ${ }^{(15)}$, lo cual podría explicar por qué el activo de ELM se solubilizó fácilmente al adicionarse a la emulsión $(\mathrm{O} / \mathrm{W})$ coincidiendo con los resultados de solubilidad que reportamos, donde el ELM es totalmente soluble en agua.

La evaluación del $\mathrm{pH}$ es uno de los parámetros usados para monitorear los cambios en la estructura de la formulación (reacciones de hidrólisis y oxidación o cambios resultantes de proceso de fabricación, como contaminación bacteriana) que a veces no son detectables visualmente y pueden afectar la calidad, eficacia y seguridad de producto final $^{(10,26)}$. Este estudio encontró el $\mathrm{pH}$ dentro del rango aceptable, de 4,0 a 6,5, para uso en la piel. El color observado fue beis, un color muy similar a la piel humana, por tal motivo esta característica podría tener una aplicación sensorial bastante aceptable en dermatología, porque, en comparación con los filtros físicos que dejan la piel algo blanquecina, esta formulación proporciona un aspecto más natural.

Como limitación del estudio se debe mencionar que el método de Mansur utiliza para el cálculo del FPS solo los valores de radiación UV de 290 a $320 \mathrm{~nm}$, sin incluir todo el rango UV, es decir desde los 200 hasta los $400 \mathrm{~nm}^{\text {(24). }}$

En conclusión, la formulación con ELM al 15\% muestra actividad fotoprotectora frente a la radiación UVB in vivo e in vitro, los alcaloides fueron los componentes fitoquímicos mayoritarios encontrados en el ELM y la crema resultó ser compatible químicamente con el activo (ELM), dando la posibilidad de usar esta formulación como agente fotoprotector solo o asociado a otros filtros solares.

Agradecimientos: Al Q. F. Armando Rivero por el apoyo en el CNCCINS para la realización de los análisis fisicoquímicos, al Dr. José Ráez (fallecido) del Instituto de Patología de la UNMSM por su apoyo de muestras histológicas, a la Q. F. Bertha Jurado (fallecida) por su ayuda en el estudio fitoquímico en la Facultad de Farmacia y Bioquímica de la UNMSM, al Téc. Lab. Juan Cueva (fallecido) por su apoyo en la liofilización del extracto acuoso de maca en la UPCH.

Contribuciones de los autores: MCA concibió y diseñó el artículo, recolectó resultados, analizó e interpretó los datos, hizo la revisión crítica del manuscrito y aprobó su versión final. CBC recolectó resultados, analizó e interpretó los datos, hizo la revisión crítica del manuscrito y aprobó su versión final. JHA recolectó resultados, hizo la revisión crítica del manuscrito y aprobó su versión final. LME contribuyó con la interpretación de datos, redacción, revisión crítica y aprobación de la versión final.

Financiamiento: La investigación fue autofinanciada por los autores.

Conflictos de interés: Los autores declaran no tener conflictos de interés en la ejecución y publicación de este artículo.

\section{REFERENCIAS BIBLIOGRÁFICAS}

1. World Health Organization. Agotamiento del ozono estratosférico, radiación ultravioleta y salud [Internet]. Geneva: WHO; 2020 [citado 15 de abril de 2020]. Disponible en: https://www.who.int/globalchange/ozone_uv/es/.
2. Schalka S, Steiner D, Ravelli FN, Steiner T, Terena AC, Marçon CR, et al. Brazilian Consensus on Photoprotection. An Bras Dermatol. 2014;89(6):1-74. doi:10.1590/abd1806-4841.20143971. 
3. International Agency for Research on Cancer. Launch of Cancers Attributable to UV Radiation [Internet]. Lyon: IARC; 2018 [citado 19 de mayo de 2020]. Disponible en: https://www.iarc.fr/news-events/ launch-of-cancers-attributable-to-uv-radiation-website/.

4. Little MP, Linet MS, Kimlin MG, Lee T, Tatalovich Z, Sigurdson AJ, et al. Cumulative solar ultraviolet radiation exposure and basal cell carcinoma of the skin in a nationwide US cohort using satellite and ground-based measures. Environ Health Glob Access Sci Source. 2019;18(1):114. doi: 10.1186/s12940-019-0536-9.

5. Watson M, Holman DM, Maguire-Eisen M. Ultraviolet Radiation Exposure and Its Impact on Skin Cancer Risk. Semin Oncol Nurs. 2016;32(3):241-54. doi: 10.1016/j.soncn.2016.05.005.

6. Servicio Nacional de Meteorología e Hidrología del Perú. Pronóstico del índice de radiación solar ultravioleta a nivel nacional. Nota técnica 002-2016-SENAMHI. Lima: SENAMHI; 2016.

7. Ministerio de Salud. Análisis de la Situación del Cáncer en el Perú, $20181^{\text {a }}$ ed. Lima: Centro Nacional de Epidemiología, Prevención y Control de Enfermedades, MINSA; 2020.

8. Gilaberte Y, González S. Update on Photoprotection. Acta Dermosifiliogr. 2010;101(8):659-72. doi: 10.1016/S1578-2190(10)70696-X.

9. Geoffrey K, Mwangi AN, Maru SM. Sunscreen products: Rationale for use, formulation development and regulatory considerations. Saudi Pharm J. 2019; 27(7):1009-18. doi: 10.1016/j.jsps.2019.08.003.

10. Piovesana de Souza F, Campos GR, Packer JF. Determinação da atividade fotoprotetora e antioxidante em emulsões contendo extrato de Malpighia glabra L. - Acerola. J Basic Appl Pharm Sci. 2013;34(1):69-77.

11. Soares Dos Reis G, Ramos Paes de Lima, Furtado Valadao A, Lucy Moreira M. Preparación de un protector solar y evaluación de la acción fotoprotectora del propóleo verde del Vale do Aço, Minas Gerais, Brasil. Boletin Latinoam Caribe Plantas Med Aromat. 2009;8(4):282-288.

12. Prudencio Quiroz JM, Bustamante Arroyo EV. Determinación in vitro de la actividad fotoprotectora UVB en una crema de protección solar formulada con extracto hidroglicólico de Lepidium meyenii (Maca) [Internet] [Tesis]. Lima: Facultad de Farmacia y Bioquímica, Universidad Nacional Mayor de San Marcos; 2018. Disponible en: http://cybertesis.unmsm.edu.pe/handle/cybertesis/7820.

13. Costa SCC, Detoni CB, Branco CRC, Botura MB, Branco A, Costa SCC, et al. In vitro photoprotective effects of Marcetia taxifolia ethanolic extract and its potential for sunscreen formulations. Rev Bras Farmacogn. 2015;25(4):413-8. doi: 10.1016/j.bjp.2015.07.013.

14. Esparza E, Hadzich A, Kofer W, Mithöfer A, Cosio EG. Bioactive maca (Lepidium meyenii) alkamides are a result of traditional Andean postharvest drying practices. Phytochemistry. 2015;116:138-48. doi: 10.1016/j.phytochem.2015.02.030.

15. Sifuentes-Penagos G, León-Vásquez S, Paucar-Menacho LM. Estudio de la Maca (Lepidium meyenii Walp.): cultivo andino con propiedades terapéuticas. Sci Agropecu. 2015;6(2):131-40. doi: 10.17268/sci. agropecu.2015.02.06.

16. Yang S, Liu D, Liu Y, Lu X, Hu F, Peng M. Analysis of total alkaloids in Meconopsis quintuplinervia from different localtites of Qinghai. Zhong Yao Cai Zhongyaocai J Chin Med Mater. 2006;29(5):430-2.

17. Spitaler R, Schlorhaufer PD, Ellmerer EP, Merfort I, Bortenschlager S, Stuppner $\mathrm{H}$, et al. Altitudinal variation of secondary metabolite profiles in flowering heads of Arnica montana cv. ARBO. Phytochemistry. 2006;67(4):409-17. doi: 10.1016/j.phytochem.2005.11.018.

18. Gonzales-Castañeda C. Efecto protector de extractos de hojas de tres variedades de Lepidium meyenii contra el daño ocasionado por la Radiación UV-B en ratones. [Tesis para optar el grado de Magister]. Lima: Facultad de Ciencia y Filosofía, Universidad Peruana Cayetano Heredia; 2009.

19. National Research Council. Guide for the care and use of laboratory animals. [Internet]. Washington D.C.: National Academies Press; 2011 [citado 14 de junio de 2020] p. 123. Disponible en: https://grants.nih. gov/grants/olaw/guide-for-the-care-and-use-of-laboratory-animals.pdf.

20. Fuentes F, Mendoza R. Guía de manejo y cuidado de animales de laboratorio: ratón. Lima: Ministerio de Salud, Instituto Nacional de Salud; 2008. Disponible en: https://www.ins.gob.pe/insvirtual/images/ otrpubs/pdf/GUIA_ANIMALES_RATON.pdf.

21. Alayo Mendoza WE, Fiestas Jacinto R, Jara CIA, Saavedra EFC. Desarrollo de una crema de hojas de piper aduncum 1 . (piperaceae) con actividad fotoprotectora in vitro. Arnaldoa. 2018;25(1):115-26. doi: 10.22497/arnaldoa.251.25107.

22. Hollands Barca IE, Gómez-Barry H, Miyares Cao CM. Modelo biológico para evaluar la acción fotoprotectora de un extracto de cordón umbilical humano. Rev Cuba Farm. 2003;37(1):20-6.

23. Choi H, Ahn S, Lee BG, Chang I, Hwang JS. Inhibition of skin pigmentation by an extract of Lepidium apetalum and its possible implication in IL-6 mediated signaling. Pigment Cell Res. 2005;18(6):439-46. doi: 10.1111/j.1600-0749.2005.00266.x.

24. Mansur J de S, Breder MNR, Mansur MC d'Ascençäo, Azulay RD. Determinaçäo do fator de proteçäo solar por espectrofotometria. Bras Dermatol. 1986;121-4.

25. United States Pharmacopeial 38. Rockville, MD: USP 38; 2015.

26. Yaringaño Moreano JM. Formulación de una crema dermocosmética a base de Mauritia flexuosa L. f. y Copaifera reticulata var. peruviana con efecto regenerador de la piel lesionada en ratones Mus musculus BALB/c. [Internet] [Tesis]. Lima: Facultad de Farmacia y Bioquímica, Universidad Nacional Mayor de San Marcos; 2015. Disponible en: https://cybertesis.unmsm.edu.pe/handle/20.500.12672/4454.

27. Inocente-Camones MÁ, Tomas-Chota GE, Huamán-Malla J, Muñoz-Jáuregui AM, García-Morán RI, Quispe-Fuentes G, et al. Actividad antioxidante y fotoprotectora in vitro de una loción y gel elaborados con extracto estabilizado de camu camu (Myrciaria dubia, Kunth). Rev Soc Quím Perú. 2014; 80(1):65-77.

28. Australian regulatory guidelines for sunscreens (ARGS) [Internet]. Therapeutic Goods Administration (TGA). Australian Government Department of Health; 2019 [citado 11 de febrero de 2021]. Disponible en: https://www.tga.gov.au/publication/australian-regulatory-guidelines-sunscreens-args.

29. Zhou Y, Li P, Brantner A, Wang H, Shu X, Yang J, et al. Chemical profiling analysis of Maca using UHPLC-ESI-Orbitrap MS coupled with UHPLC-ESI-QqQ MS and the neuroprotective study on its active ingredients. Sci Rep. 2017;7. doi: 10.1038/srep44660.

30. Sayre RM, Agin PP, LeVee GJ, Marlowe E. A comparison of in vivo and in vitro testing of sunscreening formulas. Photochem Photobiol. 1979;29(3):559-66. 